\title{
Experiencias Subjetivas de Intimidad en Pareja: Un Dilema Social Contemporáneo
}

\section{Subjective Experiences of Couples' Intimacy: A Contemporary Social Dilemma}

\author{
Andrea Rihm y Dariela Sharim \\ Pontificia Universidad Católica de Chile \\ Jaime Barrientos
Universidad de Santiago de Chile \\ Claudia Araya \\ Pontificia Universidad Católica de Chile \\ Margarita Larraín \\ Centro de Referencia de Salud Cordillera Oriente
}

\begin{abstract}
Las transformaciones culturales recientes han reconfigurado la experiencia de intimidad. Este artículo presenta y discute los resultados de un estudio cualitativo conducido en Santiago de Chile, cuyo objetivo fue explorar, mediante relatos de vida temáticos y grupos focales, los significados atribuidos a las experiencias de intimidad en la relación de pareja por 64 hombres y mujeres de entre 38 y 45 años, en un muestreo de bola de nieve. Los resultados muestran que las personas encarnan en sus relaciones de pareja las tensiones que se desprenden de la coexistencia de distintos discursos sociales sobre las experiencias amorosas. Junto con anhelar amor e intimidad, expresan sentimientos de amenaza por la dependencia, pérdida de autonomía y renuncia a los proyectos personales, que asocian a la experiencia de pareja. Se discute que abordar dichas tensiones requiere una nueva conceptualización de intimidad, que la entienda como un proceso intersubjetivo discontinuo, marcado por experiencias de ruptura y reparación. Para esto, se utiliza el concepto psicoanalítico de terceridad, que considera los vínculos de intimidad como espacios de coconstrucción fuera de la lógica de la complementariedad.
\end{abstract}

Palabras clave: intimidad, transformaciones culturales, terceridad, enfoque biográfico, relaciones de pareja

Recent cultural transformations have reshaped the experience of intimacy. This article presents and discusses the results of a qualitative study — conducted in Santiago, Chile - aimed at exploring, through life stories and focus groups, the meanings attributed to intimate relationships by 64 men and women (ages 38 to 45 ), contacted through snow ball sampling. Results show that people embody in their intimate relationships the social tensions ensuing from the coexistence of different social discourses regarding intimate experiences. While yearning for love and intimacy, they also feel threatened by the dependence, the loss of autonomy, and the giving up of individual projects that they associate with being in a couple relationship. It is argued that tackling these tensions requires a new conceptualization of intimacy, one that understands it as a discontinuous intersubjective process, marked by experiences of rupture and repair. In order to do this, the psychoanalytic concept of thirdness is used, which regards intimacy bonds as spaces of co-construction outside of the logic of complementarity.

Keywords: intimacy, cultural transformations, thirdness, biographic approach, couple relationships

La modernidad tardía se ha caracterizado — a nivel social— por el proceso de individualización, que se traduce en una organización cultural y social, tal que — a nivel individual — se ha manifestado en procesos de individuación, caracterizados por mayor libertad y autonomía para definir la propia identidad y por la

Andrea Rihm, Laboratorio Interdisciplinario de Investigación en Subjetividad y Cambio Social, Escuela de Psicología, Pontificia Universidad Católica de Chile, Santiago, Chile; Dariela Sharim, Escuela de Psicología, Pontificia Universidad Católica de Chile y Laboratorio Interdisciplinario de Investigación en Subjetividad y Cambio Social, Escuela de Psicología, Pontificia Universidad Católica de Chile, Santiago, Chile; Jaime Barrientos, Escuela de Psicología, Universidad de Santiago de Chile y Laboratorio Interdisciplinario en Subjetividad y Cambio Social, Escuela de Psicología, Pontificia Universidad Católica de Chile, Santiago, Chile; Claudia Araya, Escuela de Psicología, Pontificia Universidad Católica de Chile, Santiago, Chile; Margarita Larraín, Centro de Referencia de Salud Cordillera Oriente, Santiago, Chile.

Este estudio contó con el financiamiento de la Comisión Nacional de Investigación Científica y Tecnológica, a través de los proyectos FONDECYT N 1130860 y FONDAP N 15130009 (Centro de Estudios de Conflicto y Cohesión Social — COES).

La correspondencia relativa a este artículo debe ser dirigida a Andrea Rihm, El Bosque Sur 625, Providencia, Santiago, Chile. E-mail: arihm@uc.cl 
toma de distancia respecto de roles y sentidos institucionales organizacionalmente establecidos (Beck \& BeckGernsheim, 1990/2003; Ehrenberg, 1998/2000; Güell, Peters \& Morales, 2012). Este proceso, inicialmente descrito para sociedades industrializadas, también ha sido conceptualizado para el caso de América Latina en general, y Chile en particular, aunque con matices diferenciadores.

Chile ha experimentado una serie de transformaciones políticas y culturales que han puesto en cuestión temas que anteriormente parecían indiscutibles (Programa de Naciones Unidas para el Desarrollo [PNUD], 2015) y que han contribuido a la erosión de los discursos colectivos y a una mayor individualización de la sociedad (PNUD, 2002; Yopo, 2013). Esto último, a nivel individual, habría implicado una afirmación y defensa de la autonomía en contra de estructuras y valores autoritarios y una expansión de las expectativas de igualdad (Araujo, 2016; Güell et al., 2012), particularmente en las esferas del trabajo y la familia, que impactan profundamente las biografías (Yopo, 2013). Sin embargo, las mayores oportunidades de autodeterminación propias del proceso de individualización social han traído consigo nuevos malestares para los individuos. Estudios del PNUD $(2002,2015)$ han mostrado que se configura la imagen de un sujeto solo, que siente la presión de cumplir con estándares de éxito que parecen inalcanzables en condiciones en que no se cuenta con suficiente apoyo social y en que cada quien es su propio soporte. Se articularía, de este modo, en el discurso de los chilenos un subjetivismo biográfico que enfatiza la producción individual de las trayectorias de vida (PNUD, 2015).

En este contexto de exacerbación de las trayectorias individuales, se abre una serie de interrogantes sobre el impacto de estas transformaciones culturales en la intimidad y en los modos subjetivos de establecer vínculos cercanos. Tal como planteara Cruz Sierra (2011), "la intimidad es una experiencia en que convergen el mundo personal-subjetivo y el mundo interpersonal-social” (p. 186) y es, precisamente, esta naturaleza fronteriza la que hace que sus transformaciones sean un fenómeno de estudio interesante (Guevara Ruiseñor 2005, 2007; Sharim, Araya, Carmona \& Riquelme, 2011), aunque también complejo de definir de manera unívoca. De hecho, es difícil encontrar una definición consistente de la noción de intimidad. Guevara Ruiseñor (2005), por ejemplo, describe tres formas de intimidad reconocidas por la sociología: la intimidad como una relación cercana y profunda con otros significativos, sustentada en el mutuo conocimiento, la intimidad como un espacio privado y personal, fuera del alcance de otros, y la intimidad como un ámbito social en que se encuentran lo personal y el mundo afectivo. Costa (2006), por su parte, destaca el carácter de refugio de la intimidad, constituyendo un espacio privado y protegido de la mirada de otros, mientras que Frank, Clough y Seidman (2013) la conceptualizan como un proceso de exploración subjetiva y autorrevelación entre uno y otro, como parte de una búsqueda de autenticidad y establecimiento de un vínculo.

Si bien las relaciones de intimidad pueden darse en distintos tipos de vínculos (Frank et al., 2013), es en las relaciones de pareja en las que más se han estudiado, especialmente entre las parejas heterosexuales. En este artículo se presenta y discute parte de los resultados de una investigación cuyo objetivo fue estudiar las transformaciones de la intimidad - y su correlato de experiencia subjetiva - en las relaciones de pareja, poniendo el acento en la comprensión del lugar que se le atribuye a los otros significativos, en el contexto actual de transformaciones culturales de Chile.

Se argumenta que, en el plano de la intimidad en las relaciones de pareja, las transformaciones culturales asociadas a la individualización se han instalado de manera paradojal, coexistiendo modelos de amor opuestos entre sí, con los que las personas tratan de cumplir simultáneamente. Esto habría intensificado las tensiones asociadas a la pareja, dado que representa, al mismo tiempo, un importante anhelo y una cierta amenaza al desarrollo del proyecto personal.

\section{Los Procesos de Individualización y su Impacto en las Relaciones de Pareja}

Hace ya 25 años, Giddens (1992) observó transformaciones sociales a gran escala y de amplio efecto relativas a la individualización social. Desde su perspectiva, a nivel de las relaciones amorosas y de intimidad, la individualización se habría materializado en la evolución de un modelo de amor romántico a uno de amor confluente caracterizado por la apertura (Giddens, 1992), que no aspiraría a la fusión ni a la disolución de la individualidad, sino que promovería el desarrollo de la identidad personal. Sería contingente y opuesto a la noción de "uno y para siempre" del amor romántico: las relaciones puras, propias del modelo confluente, continuarían solo en tanto resultaran satisfactorias para cada individuo. Así, el amor confluente supondría una intimidad que resguarda la individualidad y que se diferencia claramente de la experiencia de ser absorbido por el otro (Giddens, 1992). 
Eva Illouz (2011/2012) planteó que el amor y las relaciones de pareja encarnan algunas de las transformaciones culturales más importantes del siglo XX, entre ellas, la "individualización de los proyectos de vida y la intensificación de los proyectos de vida emocionales" (p. 20). Asimismo, señaló que la mayor igualdad entre los géneros y la mayor libertad individual habrían cambiado la "textura emocional" de las relaciones de intimidad, pues las influencias conjuntas del feminismo y el discurso psicológico habrían coincidido en promover un estilo de reflexividad en que la experiencia privada, emocional, se convierte en discurso público y en que la expectativa es que las relaciones establecidas coincidan con los propios valores y preferencias, afirmando la autonomía personal (Illouz, 2006/2007). De este modo, se habría instalado en las relaciones de pareja la expectativa de crear "igualdad y un intercambio justo por medio de una implacable comunicación verbal sobre las propias necesidades, emociones y objetivos” (p. 81).

Estos planteamientos respecto de los procesos de individualización provenientes de sociedades industrializadas europeas han sido cuestionados por autores latinoamericanos (Araujo, 2005, 2009; Araujo \& Martuccelli, 2014; Benavente R. \& Vergara P., 2006; Bonan, 2005; Guevara Ruiseñor, 2005), quienes han planteado que las transformaciones tendrían otro ritmo, dada la historia latinoamericana fuertemente marcada por valores patriarcales y tradicionales (Benavente R. \& Vergara P., 2006; Guevara Ruiseñor, 2005). En el caso de México, Guevara Ruiseñor (2005) describió la situación como una "modernidad a medias" en la que "se han adoptado nuevas prácticas en un antiguo esquema de valores" (p. 874). Bonan (2005), desde Brasil, ha argumentado que, mientras en Europa el proyecto de la modernidad se articuló desde el laicismo, en América Latina la Iglesia Católica, mediante alianzas con las élites locales, ha mantenido una influencia importante en la regulación de la vida social, por lo que existiría una "recomposición reflexiva entre normas, representaciones y valores nuevos y antiguos y entre las subjetividades colectivas y las instituciones que las producen" (p. 93), en que persistiría cierto conservadurismo en el plano sexual y de género, pese a la apropiación de otros desarrollos propios de la modernidad tardía. En Chile se ha observado que la incorporación de nuevas prácticas y valores en relación a las parejas varía fuertemente dependiendo de las condiciones de vida urbana o rural del nivel socioeconómico (NSE) y del género (Benavente R. \& Vergara P., 2006; Valdés, Gysling \& Benavente, 1999). Se configura, así, un panorama que permite entender que la inclusión de los discursos de la modernidad tardía en Latinoamérica ocurriría de modo fragmentario y afectaría mayormente a algunos ámbitos de la vida, mientras que en otros persistirían prácticas más tradicionales.

Araujo y Martuccelli (2014) propusieron que en Chile la afirmación de un sentido de individualidad no estaría tan dada por el distanciamiento de los referentes y prescripciones institucionales, como en Europa, sino por el reconocimiento de la propia capacidad de hacer y enfrentar los desafíos. En este contexto, plantean que las nociones de esfuerzo personal, de alerta y apertura a las oportunidades, de carácter y de sociabilidad, adquirirían importancia y resaltan que, incluso lo relacional, tendría un tinte individual e instrum ental - además de afectivo-, pues la capacidad de tejer relaciones de apoyo sería una competencia individual. Todo esto se daría en un contexto en que las instituciones serían percibidas como una potencial amenaza al logro de las metas personales. Sus observaciones coinciden con la idea de que los individuos parecen haber empezado a buscar en el ámbito privado la seguridad ontológica y el reconocimiento subjetivo que ya no pueden encontrar en el mundo social, dotando a estos ámbitos de una particular relevancia en la definición del sí mismo y cargándolos de un peso importante (Guevara Ruiseñor, 2007; PNUD, 2002).

El estudio del impacto de las transformaciones asociadas a los procesos de individualización social sobre la construcción de vínculos de intimidad está aún en ciernes. Investigaciones anteriores (Araujo \& Martuccelli, 2012) han mostrado que las personas se sienten compelidas a estar en pareja, pero que estas relaciones aparecen cargadas de conflictos y tensiones. Se ha planteado que las relaciones de pareja -en el contexto de los procesos de individualización social - se construirían al modo de un "monólogo colectivo", en que las expectativas de vida en pareja en realidad se articulan de modo individual, en un espacio de pseudointimidad, en el que la inclusión del otro como interlocutor es "como si", pues las conversaciones son más bien soliloquios centrados en las propias expectativas, ante el temor de que incluir genuinamente al otro pudiera constituirse en una amenaza para el desarrollo de proyectos individuales (Sharim, Araya et al., 2011). Coincidentemente, los resultados de Araujo y Martuccelli (2012) resaltan que la pareja es "vista más como un campo de lucha que de encuentro" (p. 184).

Por otra parte, desde la clínica se ha planteado que la relevancia que han adquirido las relaciones de pareja, paradojalmente, las ha transformado también en un espacio potencialmente amenazante para la estabilidad subjetiva. La psicoanalista López Mondéjar (2003) ha planteado que las tendencias sociales actuales conllevan la negación de las necesidades de dependencia mutua, que son experimentadas como signo 
de debilidad. En este nuevo contexto, "la búsqueda de amor choca con las dificultades de este individualismo extremo, con la amenaza que la intimidad supone para los sujetos, con el temor a la dependencia vivida como una renuncia al proyecto personal” (p. 2510). Mitchell (1997, 2002), desde el psicoanálisis relacional, planteó que las maniobras defensivas desplegadas por las parejas para contrarrestar los sentimientos de dependencia, vulnerabilidad y agresión que inevitablemente acompañan el reconocimiento que el ser amado - de quien uno depende - son diferentes e incontrolables y estarían a la base del decaimiento del amor y de la degradación del romance en las parejas.

Goldner (2004, 2007), desde una perspectiva crítica, propone que la dependencia no sería por naturaleza perjudicial o vergonzante, sino que correspondería a una construcción social relacionada con los estereotipos asociados al género, que enmarcarían la dependencia como una experiencia asociada a lo femenino y pasivo, contraviniendo el mandato social de independencia y autodefinición. Más aún, Goldner $(2004,2007)$, tomando el concepto de terceridad que elaborara Jessica Benjamin, propuso que la dependencia podría transformarse en una experiencia de riesgo mutuo revitalizante, si se experimentara en un espacio de terceridad, es decir, como parte de un proceso intersubjetivo dinámico, no definido desde la lógica de la complementariedad y de la definición por oposición, sino desde una lógica que permita renunciar al control y aceptar las diferencias, manteniendo el vínculo. Así, conceptualizó la terceridad romántica como el contexto en el que las personas podrían sentirse suficientemente seguras para arriesgarse a depender. Esto implicaría un posicionamiento múltiple respecto a la relación, incluyendo tanto sostenerla como ser sostenido y, simultáneamente, actuar en ella como un agente individual.

Sin embargo, la idea de entregarse, renunciar al control y confiar en la relación, a pesar de las diferencias y experiencias de ruptura, está en contradicción con las tendencias culturales predominantes que sostienen el ideal - incluso el mandato - de vivir una vida de pareja y sexual "exitosa", dado que la felicidad personal a menudo se juzga a partir de ese estándar (Mitchell, 1997, 2002). Esto permitiría entender la creciente necesidad de controlar al otro para alcanzar lo anhelado, pero también las tensiones que este movimiento genera en las relaciones de pareja. Luepnitz (2002) ha criticado los mandatos culturales imperantes respecto de las relaciones de pareja, advirtiendo que las personas tienden a interpretar de manera netamente personal el fracaso, incluso cuando refleja una experiencia socialmente extendida. Desde su perspectiva, esto se relacionaría con que el discurso social predominante no aborda suficientemente cuán desafiantes y marcadas por la ambivalencia resultan las relaciones de pareja.

En este contexto, el objetivo de esta investigación fue indagar en los significados atribuidos a las relaciones de pareja por adultos chilenos de entre 38 y 45 años.

La contribución del estudio radica en considerar, en el ámbito de las relaciones de pareja, las tensiones entre intimidad y autonomía, teorizándolas en relación al concepto de terceridad y proponiendo una conceptualización de intimidad como un proceso dinámico y en continua evolución, que puede sostener y abarcar esas tensiones, tanto como los momentos de quiebre y reparación, es decir, como un proceso en que se pueden articular las necesidades de espacio personal y dependencia.

\section{Método}

\section{Diseño}

Se utilizó metodología cualitativa, con un diseño analítico relacional, para explorar y comprender los significados atribuidos por hombres y mujeres a las diferentes dimensiones de la experiencia de estar en pareja en el contexto de la propia historia. Específicamente, el estudio se enmarcó teórico-metodológicamente en el enfoque biográfico (Legrand, 1993), que considera las narrativas individuales como una vía de acceso a los significados personales sobre las experiencias vividas, en el contexto de escenarios culturales y significados compartidos, articulando dialécticamente registros sociales, microsociales y psicológicos (Correa L., 1999; de Villers Grand Champs, 1993/1999). Este enfoque considera que la exploración en profundidad de la experiencia subjetiva ofrece un camino para entender realidades sociales (Bertaux, 1980/1999; Sharim 2001), particularmente, en el contexto actual caracterizado por la individualización, en que "los cambios sociales son más visibles desde las biografías personales que desde las 'sociografías' de grupos o las clases sociales” (Martuccelli, 2010, p. 18). En el marco de una lógica ideográfica, se entiende que el estudio sistemático de la singularidad constituye una vía posible hacia un conocimiento universal (Valsiner, 2009). 


\section{Participantes}

En el estudio, realizado en Santiago de Chile, participaron 126 hombres y mujeres, abarcando dos subgrupos de edad: entre 18 y 25 años $(n=62)$ y entre 38 y 45 años $(n=64)$. En este artículo se presentan los resultados de los 64 participantes de entre 38 y 45 años (ver Tabla 1), puesto que en el caso de los jóvenes se observaron dinámicas específicas, que requerían un abordaje en profundidad (Besoain Arrau, Sharim Kovalskys, Carmona Sepúlveda, Bravo Vidal \& Barrientos Delgado, 2017).

Tabla 1

Distribución de Participantes por Sexo, Nivel Socioeconómico y Técnica de Producción de Datos

\begin{tabular}{lccccc}
\hline & \multicolumn{2}{c}{ Hombres (32) } & \multicolumn{2}{c}{ Mujeres (32) } & \multirow{2}{*}{ Total } \\
\cline { 1 - 4 } & NSE medio & NSE bajo & NSE medio & NSE najo & \\
\hline Relatos de vida & 3 & 4 & 3 & 4 & 14 \\
Grupos focales & 12 & 13 & 15 & 10 & 50 \\
Total & 15 & 17 & 18 & 14 & 64 \\
\hline
\end{tabular}

Los participantes eran heterosexuales de NSE medio o bajo y tenían educación escolar completa. La orientación sexual de los participantes se definió como criterio de inclusión para concordar con investigaciones previas sobre el tema. Por otra parte, puesto que el género ha sido considerado un factor importante en la apropiación de prácticas culturales respecto de la pareja (Benavente R. \& Vergara P., 2006; Valdés et al., 1999), se definió la necesidad de contar con un número equitativo de hombres y mujeres (32 personas de cada sexo). Asimismo, dado que existen antecedentes respecto de que la individualización se instalaría más lentamente en NSE medios y bajos (Benavente R. \& Vergara P., 2006; PNUD, 2002), el estudio se orientó específicamente a esos grupos. El NSE de los participantes se evaluó tanto en función de variables que consideraban sus condiciones de vida y ocupación actual, a partir del modelo desarrollado por ICCOM Investigación de Mercado (2005).

Los participantes fueron reclutados utilizando la técnica de bola de nieve (Patton, 2002), después de enviar una invitación abierta a través de correo electrónico a distintas personas y agrupaciones. El estatus actual de relación de pareja no se consideró como un criterio de exclusión, pues interesaba detectar las significaciones desde distintas perspectivas. Tampoco se establecieron criterios de inclusión específicos respecto de este tema, salvo que los/as participantes hubieran tenido alguna relación de pareja.

\section{Técnicas de Producción de Datos}

En el estudio se utilizaron dos técnicas de producción de datos: relatos de vida temáticos y grupos focales. Esto tuvo como objetivo tanto profundizar en distintas dimensiones de las experiencias de los participantes como triangular la información y observar a los participantes interactuando en relación a los temas de interés de la investigación (Caillaud \& Kalampalikis, 2013; Morgan \& Spanish, 1984). Como se observa en la Tabla 1, en ambas técnicas se mantuvo una distribución equitativa por sexo y NSE, pues se ha reportado que ambas variables impactan en el modo en que lo social se experimenta a nivel individual (Benavente R. \& Vergara P., 2006).

Se realizaron 14 relatos de vida, equitativamente distribuidos por sexo y NSE. Estableciéndose esa cantidad considerando que se ha planteado que una investigación con relatos de vida no debiera contar con menos de 12 participantes (Cornejo, Mendoza \& Rojas, 2008), que el número de participantes debiera permitir alcanzar la saturación de la información respecto al objeto de estudio (Bertaux, 1980/1999) y que debiera permitir captar las irregularidades, los quiebres y los detalles, no solo perspectivas globales (Sharim Kovalskys, 2005). En base a la producción y análisis de la información, se detuvo la convocatoria al concluir los relatos de 14 participantes.

Los relatos de vida se entienden como la narración que un sujeto hace de su vida o de parte de esta (Cornejo, 2006). En este caso, los relatos de vida temáticos se focalizaron específicamente en la historia de pareja, utilizando la consigna "Cuéntame tu historia de pareja". Como ha planteado Arfuch (2010), al 
investigar usando relatos de vida, el objetivo es buscar una historia que se genera en el diálogo del proceso de investigación. Esta mirada enfatiza la naturaleza dinámica y relacionalmente situada de los relatos de vida, al mismo tiempo que da a los participantes la posibilidad de construirse y reconstruirse en el ejercicio de narrar, ofreciendo un espacio de reflexión (Cornejo, 2006; Sharim, Kovalskys, Morales \& Cornejo, 2011). Se entiende, entonces, que la narración de la propia historia es una expresión identitaria, por el trabajo de construcción y reflexión sobre sí mismo. Es precisamente en esta acción del sujeto donde puede observarse la articulación de lo social y de lo individual, espacio central para la discusión de las hipótesis que aquí se manejan sobre la experiencia de relación con otro-pareja (Digneffe, 1995; Ferrarotti, 1981/2013; Lainé, 1998).

Como parte del estudio, se realizaron 14 grupos focales. De estos, ocho — cuyos resultados se reportan en este artículo- fueron con participantes entre 38 y 45 años. Los grupos focales tuvieron como objetivo aproximarse a las narrativas sociales sobre las relaciones de pareja, considerando que las interacciones grupales estimulan a los participantes a explorar y clarificar perspectivas, tanto individuales como compartidas (Tong, Sainsbury \& Craig, 2007). Los grupos focales proveen, además, de información acerca de un conjunto amplio de ideas y sentimientos que las personas pueden tener sobre un tema, así como iluminar las diferencias existentes sobre un determinado tema entre individuos pertenecientes a grupos diversos, en este caso, hombres y mujeres (Rabiee, 2004).

Los grupos focales estuvieron compuestos por entre 4 y 8 personas (moda 7). De los ocho grupos, se definió que dos de ellos fueran mixtos y los seis restantes estuvieran compuestos solo por hombres o solo por mujeres. Finalmente, los grupos contaron con la participación de 25 personas de cada sexo.

\section{Procedimiento}

Se realizaron dos encuentros con cada participante de los relatos de vida, de entre 45 y 106 minutos cada uno, en lugares y momentos convenientes para ellos. Durante los encuentros, los/as entrevistadores/as —ocho mujeres y un hombre, con formación de pre y postgrado en psicología-intervinieron mínimamente, solo para clarificar dudas.

Dos de los grupos focales se realizaron en consultas de miembros del equipo de investigación y seis, en las dependencias de SUR. Fueron conducidos por una dupla de psicólogos del equipo de investigación, cautelando que al menos uno de los conductores del grupo fuera del mismo sexo que los participantes. Así, dos fueron conducidos por dos hombres, tres por dos mujeres y tres por una dupla mixta. La duración promedio de los grupos fue de 1 hora y 36 minutos. Inicialmente se pidió a los participantes asociar un color con las palabras amor, intimidad y sexualidad. Esto se usó como un estímulo para generar la conversación, marcando las diferencias y similitudes emergentes entre las respuestas (e.g., en términos de los colores predominantes). Luego, la conversación fluyó sustentada en los contenidos presentados por los participantes en relación a sus experiencias de pareja.

El proyecto fue aprobado por el Comité de Ética de la Escuela de Psicología de la Pontificia Universidad Católica de Chile. Para resguardar la voluntariedad de la participación se envió una carta de invitación que describía el estudio y entregaba información de contacto de la investigadora responsable en el caso de que los potenciales participantes quisieran clarificar algún aspecto. En el momento del encuentro/grupo focal se presentaron los consentimientos informados, que especificaban la voluntariedad de la participación. Los participantes los firmaron, aceptando el uso de grabaciones y transcripciones. Para resguardar la confidencialidad de los participantes, se eliminaron de las transcripciones los datos que pudieran resultar identificadores y se sustituyeron sus nombres por pseudónimos.

\section{Análisis de la Información}

Se utilizaron progresivamente dos lógicas de análisis del material (Cornejo et al., 2008; Legrand, 1993; Sharim Kovalskys, 2005). Primero, se realizó un análisis fenomenológico-hermenéutico en profundidad de cada relato, utilizando una lógica singular, buscando en las producciones individuales la marca o el sello de los discursos sociales disponibles (Molitor, 1990; Starks \& Trinidad, 2007). Luego, siguiendo una lógica transversal, se analizaron los relatos en su conjunto, extrayendo dimensiones o categorías centrales y determinando ejes de análisis respecto de la experiencia de intimidad. Para esto se realizó un análisis cualitativo de contenido, basado en las propuestas de Bardin (1977/2002) y de Andréu Abela (1998). Los grupos focales se analizaron siguiendo las mismas lógicas, pero incluyendo también en el análisis las formas de interacción entre los participantes y los acuerdos y divergencias emergentes en las narraciones. 
Con el objetivo de triangular perspectivas de análisis del material y enriquecer el proceso analítico (Flick, 2002/2004), este incluyó una serie de dispositivos. Entre el primer y segundo encuentro con los participantes se realizaron sesiones de inter-análisis en duplas (Cornejo et al., 2008) con foco en los contenidos emergentes, tanto como en la interacción y la relación de investigación. Este inter-análisis fue la base para construir cuadernos reflexivos. Para los grupos focales, los investigadores a cargo de cada grupo desarrollaron un cuaderno reflexivo conjunto. Posteriormente, cada cuaderno reflexivo fue analizado y discutido en el equipo de investigación (12 personas), quien había leído las transcripciones. En el caso de los relatos, el acento del análisis en equipo fue la escucha de la historia, sus resonancias y pistas para el análisis transversal, mientras que en el caso de los grupos focales, además de la experiencia de la escucha del grupo, se abordaron las dinámicas de interacción y las claves temáticas para el análisis respecto de los significados atribuidos a la intimidad, amor y sexualidad.

Estas instancias fueron clave para promover un ejercicio de reflexividad respecto a cómo las propias posiciones subjetivas de los investigadores impactaban en el modo de aproximación al material y a los participantes (Etherington, 2004), especialmente en el contexto del enfoque biográfico, que considera el carácter intersubjetivo, interdependiente y recíproco de la relación de investigación, en que "no se puede conocer sin ser removido en lo personal, sin de alguna manera transformarse" (Sharim, Araya et al., 2011, p. 86).

\section{Resultados}

El material mostró que las relaciones de pareja siguen siendo significadas como relevantes, pero que son experimentadas como marcadas por una serie de experiencias disímiles y difíciles de articular entre sí: dificultades, complejidad, tensión y ambivalencia conviven con compañía, aceptación, reconocimiento, entrega y crecimiento.

Una de las principales recurrencias en los relatos de los participantes (hombres y mujeres) fue la experiencia de no saber, de confusión y falta de certidumbre respecto de los espacios de intimidad en las relaciones de pareja. Se mostraron inseguros sobre su lugar en las relaciones de pareja, sobre cómo ser hombres y mujeres en este tiempo y sobre cómo conciliar deseos y expectativas, a menudo contradictorios entre sí.

Se observó en los participantes una oscilación entre visiones más escépticas y otras más románticas de las relaciones de pareja; entre el anhelo de estar en pareja y el temor a perder la propia individualidad en el otro. Puestos en el contexto de multiplicidad de referentes y modelos disponibles sobre el amor y las relaciones de intimidad, los participantes mostraron sentirse a la deriva entre los distintos modelos, tratando de conciliarlos y sostenerlos simultáneamente.

Puesto que esta recurrencia se observó sin mayores diferencias a nivel de las narraciones individuales y colectivas, los resultados no se presentan diferenciados en función de las técnicas de producción de datos. Sin embargo, en ocasiones la profundidad con que los participantes se adentraron en los temas varió, adquiriendo los relatos de vida un carácter más personal y centrado en la historia familiar, mientras que en los grupos focales se dio más énfasis a la crítica social.

Sí se observaron algunas diferencias de género a nivel del posicionamiento respecto de los referentes de género tradicionales, por lo que en la presentación de resultados se establecen diferencias en este sentido, aunque se parte presentando los hallazgos comunes para hombres y mujeres.

\section{El Temor a la Dependencia y la Afirmación del Proyecto Personal}

$\mathrm{Al}$ caracterizar sus experiencias de relación de pareja, la mayoría de los participantes destacó el nivel de exposición que las relaciones implican y los sentimientos de vulnerabilidad y fragilidad que la exposición ante otro conlleva:

Cuando estai en pareja tenís que sacarte la armaduras, las máscaras, tenís que dejar todo eso de la puerta pa'

fuera. Por dentro somos todos niños... (hombre, GF 1, hombres) ${ }^{1}$

Esta vulnerabilidad, inherente a la exposición al otro, aparece caracterizada como un arma de doble filo: necesaria para alcanzar conexión y aceptación, pero temida, pues conlleva el potencial de ser herido y dañado,

\footnotetext{
${ }^{1}$ Se señala el sexo del participante, si participó en relato de vida o grupo focal (GF) y, si tal es el caso, en qué tipo de grupo: mixto o no.
} 
especialmente ante la anticipación de conflictos que pudieran llevar al término de la relación. Aparece, entonces, en los participantes una fuerte valoración de la independencia, incluso al interior de las relaciones:

Yo..., muchas veces, cuando estamos con mi pareja, yo le digo “¿Cachai [entiendes] que si tú te vai, yo me voy, yo sigo mi vida?”. Y me dice: “¿Por qué?”. "Porque yo sigo haciendo todo, no dependo de ti, no dependo de mis hijos, no dependo de nadie, yo siempre he sido independiente, tengo mi espacio y me gusta ser independiente". (hombre, GF 14, hombres)

Los participantes relacionan el temor a la dependencia y la vulnerabilidad con experiencias previas de fracaso y sufrimiento, pero también con una clara consciencia de que el mundo de las relaciones de pareja ha cambiado rápidamente y que mientras generaciones anteriores "aguantaban" y seguían juntas a pesar de los conflictos, ese modelo ya no es viable. Así, los participantes, mayoritariamente, expresaron una visión fragilizada de las relaciones de pareja, en las que la posibilidad de una ruptura se instala desde el inicio y en que no hay nadie a quién responder además de a sí mismos:

Antiguamente, lo que dijo no sé quién, los matrimonios duraban eternamente ¿Y por qué duraban? Porque se aguantaban muchas cosas, infidelidades, entonces, era como "No, tú tienes que aguantarle". (mujer, GF 13, mixto)

Una vez estaba viendo un reportaje de un..., no sé cómo se llama..., un programa cultural nacional y entrevistaban a un matrimonio que llevaba 45 años juntos y este compadre, este viejo, salía todos los miércoles a una casa de putas, a sabiendas de la señora, y el viejo llegaba de la contru [construcción], se emperifollaba bien, se peinaba..., quedaba lindo, corbatita... y se iba a una casa de putas todos los miércoles, sagrado, durante 45 años de matrimonio. Y a la señora le preguntaban: “¿Y usted acepta eso? Y ella decía: "Ay sí, lo que pasa es que él es bueno conmigo". (hombre, GF 1, hombres)

Si bien un número significativo de participantes expresó cierto desdén respecto de los referentes de género que tradicionalmente regulaban las relaciones de pareja, reconocieron que el declive de estos los ha enfrentado a una situación nueva frente a la cual cada quien debe desarrollar sus propias estrategias, sin referir a modelos alternativos que jueguen un rol ordenador equivalente. En este contexto, son la individualidad y la preservación de los propios intereses las nociones que se instalan en un lugar central: la pareja tiene sentido en tanto ofrezca un espacio satisfactorio para cada individuo:

Siempre hemos conversado los dos y siempre le digo: "El día que dejís de, dejís de, que yo te deje de sentir como compañera, que nos apañamos. ¿Cachai [entiendes]? Los dos, chao, o sea, no tengo nada que hacer acá”. (hombre, GF 14, hombres)

En este sentido, se observó entre los participantes un intento por poner las relaciones de pareja en un lugar secundario, para no depender de la pareja como un proyecto único y central para alcanzar la propia felicidad:

Mi sueño fue casarme, tener vaquitas, chanchitos en el campo. ¡Ese era mi sueño! Pero con los años uno, aquí en Santiago, como que le meten el bicho de estudiar y hacer hartas cosas, y para mí la felicidad más grande ha sido estudiar. El venir a tomar un libro, entonces, entrar a la universidad, conocer cosas nuevas que yo no conocía, aprender otros conocimientos, compartir con mis compañeros, yo no siento, el, el, el ser feliz al tener un hombre al lado [...] ¡Es que hay una cosa que me llena más que el hombre! La paso mal, la sufro, me las lloro con mi mamá si me va mal en un ramo, pero... jes que sufro más que con una pérdida de un hombre! (mujer, GF 10, mujeres)

Esta preocupación por resguardar el proyecto personal contrastó con la percepción de un número importante de participantes respecto de la baja ponderación que tenía el proyecto individual en generaciones anteriores, lo que cuestionan y critican fuertemente, mostrando cuán importante es para ellos diferenciarse de las prácticas relacionales propias de las de otras generaciones.

\section{El Miedo a Ser los Hombres y Mujeres del Pasado}

Si bien los hombres y mujeres del estudio expresaron cuánto los atemorizaba repetir patrones relacionales y de género del pasado, las razones a la base de sus temores fueron diferentes. En la mayoría de las mujeres la relación con los hombres apareció teñida por suspicacia, por el temor de ponerse a sí mismas en un lugar de sumisión y por una actitud de rebelión respecto de nociones tradicionales que pudieran atarlas al pasado. En el caso de los hombres, el temor fue a seguir siendo "brutos" como sus padres y abuelos, desconsiderados, violentos y dañinos en el trato con las mujeres. Estas diferencias se desarrollan a continuación.

Mujeres que intentan cuidar de sí mismas. Entre las mujeres del estudio, tanto a nivel grupal como individual, se escuchó una fuerte crítica a las generaciones anteriores, a sus padres poco sensibles y 
machistas, pero también a sus madres, muchas de las cuales aparecen retratadas como sumisas e incapaces de poner límites a sus parejas. Esto fue particularmente marcado entre quienes crecieron en familias que adscribían a una distribución más tradicional de roles de género y contrastó con la experiencia de quienes habían tenido madres profesionales y con un proyecto de desarrollo personal propio. En este sentido, las mujeres del estudio sistemáticamente pusieron la relación de pareja en un lugar secundario, después de sus proyectos personales y de la maternidad, ya fuera en oposición a sus madres o profundizando el camino inicialmente abierto por ellas:

Ella ya no se lo permitió y se resignó no más. Y eso fue lo que yo no quise [...]. No me interesa lo que digan, a mí nadie me, me, me mantiene, soy una mujer independiente y, y yo veré lo que hago. "Pero yo no quiero, mamá, ser como usted, no quiero, me niego a eso, no quiero". Porque yo veía una mujer servil, nada más, nada más que eso. (mujer, GF 4, mujeres)

Mi mamá, eh, también estudió en la universidad y también yo creo que ella ha vivido, o sea, yo creo altos y bajos [...]. Ella proyectó ciertas cosas en mí, por ejemplo, eso de "si quieres casarte, te casas, si no, no te casas, si quieres tener hijos, ten hijos, si no, no". Porque yo creo que a ella efectivamente la guiaron hacia como que tenía que casarse, que tenía que tener hijos, que tenía que tener esta vida, y de hecho, yo creo, que está con mi papá, no porque está enamorada, sino porque ella tenía que estar al lado de mi padre. Porque así le enseñaron a ella, pero ella no me enseñó eso a mí. (mujer, GF 4, mujeres).

Parte importante de las mujeres del estudio coincidió en reconocer que crecieron con mayor libertad que sus madres, pero también que heredaron parte de su temor, sabiendo que estos espacios de libertad aún no están completamente consolidados. Para contrarrestar este temor, tendieron a adoptar posiciones de mayor control en las relaciones, tratando de resguardar su individualidad de los posibles embates de los demás, limitando cuánto entregan y cuánto ceden e intentando comunicar abiertamente sus necesidades:

Y yo creo que tiene que ver con un poco de egoísmo que a veces la gente te critica, no sé si egoísmo es la palabra, pero sentir, pensar en ti más que en el otro. ¿Cachai [entiendes]? Porque uno siempre tiende a ceder, ceder, ceder todo el rato, entonces no, po', yo ya no quiero, no estoy dispuesta ahora, o sea, por ningún motivo. (mujer, GF 7, mixto)

Me presento en forma consciente en la vida para decir lo que quiero, lo que necesito, lo que me gusta de ti y lo que no me gusta de ti. Expre..., expresar las cosas. (mujer, GF 2, mujeres)

Parte significativa de las mujeres del estudio se mostró asumiendo la labor de cuidarse a sí mismas, de responsabilizarse por el cumplimiento de sus proyectos personales, intentando no depender de nadie, ante la posibilidad de que eso implicara sumisión. De este modo, valoran la posibilidad de estar en pareja y lo que esto podría aportarles, pero han tendido a tomar distancia de la necesidad de estar en pareja, como reafirmación de su propia autonomía.

Hombres buscando un lugar. En su mayoría, los hombres del estudio también fueron críticos de las generaciones precedentes. Por ejemplo, con su deseo de no ser "brutos" como sus antecesores, mostraron su intento de relacionarse de un modo distinto con las mujeres, poder responder a sus demandas y estar más conectados emocionalmente. Sin embargo, en muchos casos sus historias familiares parecen no ofrecerles referentes para enfrentar este desafío y eso les resulta incómodo:

Y él [abuelo] me marcó. Él me marcó harto..., se casó cuatro veces..., entonces, su segunda señora murió. La cuestión es que se casaron, pero sin amor, absolutamente funcional, para el cuidado de los niños... ¿Cachai [entiendes]? Y mi abuelo por fuera siempre con otras mujeres [...] Yo tengo una ética contemporánea, entonces, entro en conflicto. Claro [ríe] él [abuelo] en su ética, estaba perfecto. Pero claro, yo creo que ahí hay un rollo [conflicto]. Interesante, interesante, sí, claro, porque la lucha de uno es no ser infiel. (hombre, relato de vida)

Desde esta ética contemporánea sobre la masculinidad, para la cual muchas veces carecen de referentes cercanos, los participantes expresaron su frustración respecto de la demanda que les hacen las mujeres en cuanto a que se relacionen de un modo diferente:

Ella conversa mucho, mucho, mucho, pero se le desbordan las palabras, tiene una gran... inteligencia emocional y yo soy bruto, torpe y... y... acostumbro a guardar en el baúl mis..., todas mis cosas las guardo en el baúl. Cada cierto tiempo... abro el baúl y empiezo a salir de lo acumulado, del baúl [...]. O sea, así, pa, pa, pa [onomatopeya de golpeado]. Porque..., claro, como no soy como ella, eh, ella es como la administradora de la emoción y yo no. Yo soy el torpe bruto que lanzo a la primera, así los primeros cuchillos los lanzo yo y, claro, después tengo que morderme la lengua, pedir disculpas [...] Yo me tengo que disculpar... porque... porque yo dejo la cagada. (hombre, GF 12, hombres)

Gran parte de los participantes hombres declaró sentirse en situación de desventaja con las mujeres en el nuevo contexto de relación. En sus relatos las mujeres aparecen dominando el terreno afectivo y emocional, 
capaces de expresar sus necesidades; mientras que ellos se sienten menos hábiles para expresar verbalmente lo que desean y necesitan. Esto, sumado a la liberación que observan en las mujeres, los hace sentirse en una posición particularmente frágil, que los expone en mayor medida que antes al abandono:

Lo que pasa es que ahora las mujeres son profesionales y son liberadas, o sea, antes teniai que... si te manteniai tan bien en un matrimonio es porque estabai... ¿Qué hacís si te separai? "No tengo plata, él me mantiene". En cambio una mujer ahora es profesional y dice: "Si no me gustai, chao". Y cambian [...]. El hombre igual siente de alguna forma esa presión de ver a una mujer que también es exitosa, que también tiene plata, que también tiene, no sé, tiene expectativas, que antes no era así ¿Cachai [entiendes]? Entonces, el hombre también tiene que adecuarse a esas cosas, o sea, el hombre también tiene que adecuarse a esas cosas, o sea, que le dé vergüenza... a lo mejor su mujer tiene más estudios que él... y gana más plata que él ¿Cachai [entiendes] o no? Entonces, ahí la mujer... tú pensai: "Oye, pero la mujer podría pescar a un hombre y mandarlo pa' la calle". (hombre, GF 1, hombres)

En los relatos de hombres las mujeres aparecen en una posición de poder que instala con mayor fuerza la necesidad de responder a sus demandas y expectativas. Es así como, en el caso de los hombres, la visión respecto del pasado combina elementos de fuerte crítica con un cierto tinte de nostalgia por la seguridad perdida a partir de las transformaciones culturales. En este sentido, los participantes revelaron una posición conflictuada respecto de sus roles y el lugar en el que pueden sentirse cómodos y seguros en una relación de pareja.

\section{La Persistencia del Anhelo de Amor e Intimidad}

Aun cuando los resultados anteriores dan cuenta de una experiencia más bien sombría y conflictuada respecto de las relaciones de pareja, esta no es unívoca. Los participantes aparecieron tensionados entre expectativas tradicionales e ideas progresistas de cómo debieran ser las relaciones de pareja.

Este conflicto se manifestó en una actitud ambivalente hacia el amor y el romance. Los relatos mostraron que el discurso romántico aún persiste, aunque no es fácilmente admisible. Por el contrario, aparece "a pesar de sí mismos", ligado a una actitud igualmente crítica y escéptica respecto del amor, marcada por el desencanto y desilusión con las relaciones de pareja. De hecho, un número importante de participantes usó las entrevistas y los grupos focales para compartir preguntas como “¿Qué he hecho mal?”, reflexionando sobre cuán distintas han resultado ser sus historias de pareja respecto de lo que habían imaginado. La coexistencia de discursos sociales contradictorios fue considerada clave por los participantes para entender esta brecha que sentían:

Agradezco haber nacido a este lado del mundo y en esta época, porque tengo todo el permiso y toda la facilidad del mundo para meterme con quien yo quiera, para elegir lo que yo quiero cada día de mi vida [suspiro]. Y, sin embargo y a pesar de ello, y por haber leído todos los cuentos de princesa cuando era chica [...], me pasa que, aun en el fondo de mi corazón..., espero cierto tipo de amor... puro y recíproco, porque esta cosa de meterse con uno, con otro, de probar, la infinidad de pasteles [inmaduros] que hay allá afuera [...], me cansa, me cansa, es un tema que me aburre. (mujer, GF 4, mujeres)

Esta viñeta refleja la incomodidad hacia las relaciones de pareja compartida por buena parte de los hombres y mujeres participantes. Sus relatos expresaron una mezcla de emociones, incluyendo cierta rabia difusa hacia el mundo, por no entregarles claridad, y hacia ellos mismos, por desear cosas contradictorias. Así, aunque superficialmente, los participantes parecen haberse adaptado a los nuevos modos de relación, dudan de su real valor y anhelan algo más. Particularmente, la mayoría de los hombres expresó la esperanza de encontrar aceptación, reconocimiento y apoyo emocional en sus relaciones de pareja:

Lo que tú querís es un momento de ternura, querís cariño, querís aceptación, querís complicidad... claro... que te apapachen. (hombre, GF 1, hombres)

Sin embargo, estos anhelos conviven con recuerdos de experiencias previas dolorosas, de rupturas, que determinan que la mayoría de los participantes se muestre simultáneamente anhelante y escéptico respecto del amor y la intimidad, limitando su apertura a arriesgarse nuevamente:

Me cuesta más transar ahora. Yo creo que trancé mucho, de repente, en mis otras dos relaciones [...]. Entonces, ahora como que... como que prendo la alarma antes que nada. También ahí es difícil, porque no dejai que nada... no... yo no dejo que haya mucha intimidad, y tengo unas relaciones como... más puertas afuera, que no se involucren mucho. (mujer, GF 2, mujeres)

Yo, por lo menos ahora, tengo más miedo que cuando joven, pienso un poquito más las cosas, con respecto a involucrarme, involucrarme en pareja, involucrarme por [...]. Yo creo que por el fracaso de mi matrimonio... el fracaso en el sentido de que, de que me divorcié, lo cual nunca quise Yo, cuando me casé, me casé para toda la vida. (hombre, GF 12, hombres) 
Los participantes expresaron que, tras fracasar en sus relaciones previas, estaban menos dispuestos a comprometerse y que protegían más su individualidad. Aquellos que sostenían una posición más escéptica hacia el amor y la intimidad, compartían el supuesto de que las relaciones de pareja no son conciliables con el logro personal, sino más bien opciones excluyentes y que estar en pareja representa más una carga que una oportunidad.

Sin embargo, en esta oscilación entre escepticismo y esperanza, el escepticismo no fue absoluto. De hecho, muchos participantes fueron capaces de reconocer en sí mismos esta tensión, mostrando un discurso crítico de quienes tenían anhelos románticos, pero simultáneamente experimentándolos ellos mismos:

Yo estereotipaba a las personas. Como que yo decía: “Ah, no sé qué, yo deseaba esto [tono irónico]”. Y la verdad es que yo tengo ganas de tener una pareja también, tengo ganas de rehacer mi vida, de, de tener a alguien [suspiro]. Sobre todo porque me gusta, yo, eh, coincido con que uno lo pasa bien en pareja, uno sale, quieres salir a comer un día o tienes ganas de no sé o vas a ir a trotar o quieres ir a, no sé, de picnic al parque y tienes alguien que te acompañe y conversas [...]. Entonces, yo, por ejemplo, ahora, yo me saqué el estereotipo de encima [...]. Es simplemente quiero [...] que me guste una persona y quiero querer, quiero amar y que me amen. (mujer, GF 4, mujeres)

Los resultados mostraron que los participantes asumen una posición ambivalente respecto de las relaciones de pareja: anhelan el reconocimiento, pero temen la dependencia; valoran la compañía, pero se rebelan ante la posibilidad de que esta sea a costa de sus proyectos personales; desprecian los patrones relacionales de género del pasado, pero se muestran incómodos con su posición de hombres y mujeres contemporáneos y, en ciertas situaciones, parecen desear un tipo de amor romántico más propio del pasado.

\section{Discusión y Conclusiones}

Los resultados dan cuenta de una transformación en los modos de construir intimidad en las relaciones de pareja, que aparece atravesada por los procesos de individualización social. En el escenario actual, no es suficiente definir la intimidad como refugio, como fuera caracterizada a principios del siglo XX (Guevara Ruiseñor, 2005), pero tampoco es suficiente homologarla a la dependencia (López Mondéjar, 2003). La noción de intimidad que se desprende de los modos actuales de relacionarse en pareja supera esos límites, al encarnar simultáneamente una serie de experiencias disímiles y contradictorias entre sí. Por momentos, sigue siendo el lugar de resguardo y privacidad que protege del mundo externo y donde es posible encontrar reconocimiento subjetivo (Guevara Ruiseñor, 2005), pero también es un lugar amenazado y amenazante, que podría atentar contra la consecución de los proyectos individuales. En este sentido, la experiencia de intimidad en las relaciones de pareja parece estar marcada ineludiblemente por una tensión.

La posibilidad de sostener esa tensión estuvo mayormente ausente del relato de los participantes, quienes tendieron a describir sus experiencias en términos binarios, moviéndose entre polos contradictorios entre sí, dando cuenta de una dificultad importante para articular sus necesidades de autonomía y dependencia. Este conflicto entre autonomía y dependencia fue percibido por los participantes como desestabilizante y potencialmente conducente a la ruptura de las relaciones. La disposición a "no transar" para resguardar el proyecto personal dio cuenta de una dificultad para flexibilizar su posición subjetiva y moverse situacionalmente entre posiciones más autónomas y más dependientes.

En las mujeres el temor a revivir la sumisión del pasado y en los hombres el temor a ser avasallados por las mujeres "dueñas" de la palabra y del manejo emocional, estuvo continuamente presente, traduciéndose en estrategias de evitación del conflicto, pues si este se hacía explícito, la ruptura se volvía una realidad inminente. Por otra parte, cuando los conflictos se explicitaban, eran experimentados intensamente, desde la posición de defensa personal ante la posibilidad de ser arrasados por el otro. Así, incluso temas que pudieran haber sido considerados mínimos, se transformaban en un espacio de afirmación de la posición subjetiva. Esto coincide con la descripción de Araujo y Martuccelli (2012) de las relaciones de pareja como un espacio de lucha y podría relacionarse con los procesos de individualización social, pues, al transformarse el individuo en una meta normativa (Araujo, 2009), su defensa se vuelve imperiosa. La expectativa de reconocimiento parece hipertrofiarse y no admitir sino una aceptación total y absoluta de sí, que deja poco espacio para el otro, aun cuando, tal como nota Illouz siguiendo a Butler, "el reconocimiento empieza con advertir que se está perdido en el otro, apropiado en y por una alteridad que es y no es uno mismo" (Butler, 2006, p. 340, citada en Illouz, 2006/2007, p. 91).

En relación con lo anterior, los resultados muestran cuán difícil resulta para los participantes construir una terceridad romántica (Goldner, 2007), esto es, un espacio que pueda contener tanto las experiencias 
positivas como de ruptura, permitiendo que la experiencia romántica gane densidad afectiva y dé cuenta de la historia de la relación. Como ha planteado Goldner (2004), la intimidad en las relaciones de pareja se nutre de las tensiones entre reconocimiento mutuo y negación, destrucción y reparación, éxito y fracaso. De este modo, es posible entender que si las experiencias de quiebre no se toleran como parte de la relación, sino que se evitan o desencadenan quiebres, la intimidad se empobrece. Esto permite dar cuenta de los resultados de una investigación anterior (Sharim, Araya et al., 2011) en la que se planteó la existencia de una pseudointimidad entre los participantes.

Estos resultados permiten entender que la existencia de intimidad es clave para que la experiencia de riesgo y dependencia sea tolerable por las parejas, pero, a su vez, que la intimidad requeriría un cierto tipo de vínculo: uno donde los quiebres son esperables y reparables, en un espacio de terceridad. De esta manera, es posible plantear una relación de influencia mutua entre terceridad e intimidad, en la que la existencia de intimidad psicológica reflejaría la presencia de terceridad y en la que el empobrecimiento de la intimidad impactaría en la posibilidad de construir un espacio de terceridad que, a su vez, permitiera tolerar la dependencia, la pérdida de control y los quiebres.

Otro de los resultados es que los participantes usan tanto nociones románticas como confluentes para describir sus experiencias de intimidad, tal como fue descrito por Giddens (1992). Más que renunciar a las expectativas tradicionales, las yuxtapusieron con nociones más postmodernas del amor, expresando la tensión que encuentran en la cultura, dando cuenta de una apropiación parcial de los modelos de amor imperantes. Esto es significativo, dado que los participantes eran de NSE medio y bajo, lo que aporta evidencia en contra de la hipótesis que los discursos postmodernos no han permeado aún los grupos sociales menos privilegiados (Benavente R. \& Vergara P., 2006).

La idea de intimidad como terceridad podría facilitar la comprensión del sentido que tiene la yuxtaposición de discursos evidenciada por los participantes, pues permitiría entender que las relaciones no pueden sostenerse solo en el romanticismo (que implica subyugar el proyecto personal al proyecto común) ni tampoco en la satisfacción temporal de las necesidades personales. Si la intimidad en las relaciones de pareja es entendida como un proceso dinámico y discontinuo, los miembros de la pareja pueden moverse a destiempo o al unísono entre posiciones más románticas y confluentes, teniendo el espacio de terceridad como una base para tolerar la ansiedad de sentirse vulnerables e inseguros respecto del futuro de la relación. La intimidad requeriría de una articulación creativa entre las necesidades de autonomía y dependencia e incorporar la noción de terceridad a la comprensión de las relaciones de pareja ayudaría a trascender los discursos que oponen autonomía y construcción de un proyecto común, pues el supuesto de que los espacios personales son necesarios y enriquecen la relación subyace a la noción de terceridad.

Los tiempos actuales conducen a un continuo cuestionamiento del sí mismo y de las relaciones, permitiendo la emergencia de nuevos discursos sobre la intimidad (Brown, 2005). Una conceptualización de la intimidad debiera incluir el espacio de lo desconocido e incierto para capturar su naturaleza dinámica.

Los resultados permiten concluir que efectivamente los participantes significaron sus experiencias de pareja de un modo que refleja - y por momentos discute con- los procesos de transformación cultural e individualización social experimentadas por Chile durante las últimas décadas (Barrientos \& Páez, 2006). Así, es preciso resaltar la importancia de comprender las tensiones experimentadas por los participantes no de modo privado o como fruto de una deficiencia individual, sino como expresión de los determinantes sociales a los que están expuestos y que ofrecen "retazos" de sentido (PNUD, 2002) con los cuales deben intentar articular modelos y referentes a menudo contradictorios entre sí.

La experiencia de relación de pareja y los modos actuales de significarla dan cuenta de una noción de los vínculos de intimidad altamente tensionada, que oscila entre el anhelo de la cercanía y la percepción de amenaza al proyecto personal que esta implicaría.

Dada la complejidad de las relaciones de intimidad y la riqueza del material aportado por los participantes respecto de sus experiencias de pareja, los hallazgos presentados no pretenden abarcar todas las dimensiones de análisis posibles. Si bien esto constituye una limitación, futuras publicaciones podrían profundizar en las diferencias en relación al género, el NSE y la generación.

También cabe pensar que otra de las limitaciones del estudio es que todos los participantes fueron heterosexuales, pese a que se ha reconocido que entre las principales transformaciones de la modernidad tardía se encuentra la liberalización de la sexualidad y aceptación de las relaciones de pareja del mismo sexo (Giddens, 1992). Estudios recientes en Chile muestran que actualmente se observan actitudes menos 
homofóbicas de la sociedad, lo que se ha traducido en mayor acceso a derechos para gay y lesbianas (Barrientos Delgado, 2015). Esto, sumado al análisis de los resultados preliminares, implicó que durante el curso del estudio se cuestionara la orientación heterosexual como criterio de inclusión en el estudio e hizo visible la necesidad de ampliar la mirada. Con este propósito, se realizaron nuevas entrevistas y grupos focales para abordar las experiencias de parejas del mismo sexo y explorar si existen - $\mathrm{o}$ no- diferencias significativas con parejas heterosexuales.

\section{Referencias}

Andréu Abela, J. (2002). Las técnicas de análisis de contenido: una revisión actualizada. Sevilla, España: Centro de Estudios Andaluces. Extraído de http://public.centrodeestudiosandaluces.es/pdfs/S200103.pdf

Araujo, K. (2005). Vida cotidiana y transformaciones de género: la esfera doméstica. Revista de La Academia, $10,77-117$.

Araujo, K. (2009). Individuo y feminismo. Notas desde América Latina. Íconos: Revista de Ciencias Sociales, 33, 141-153.

Araujo, K. (2016). Desigualdades interaccionales e irritaciones relacionales: sobre la contenciosa recomposición del lazo social en la sociedad chilena (Serie Documentos de Trabajo COES. Documento de trabajo $\mathrm{N}^{\circ}$ 3). Santiago, Chile: Centro de Estudios de Conflicto y Cohesión Social. Extraído de http://coes.cl/wp-content/uploads/2016/07/DTN\%C2\%BA03-Desigualdades-interaccionalese-irritaciones-relacionales-.pdf

Araujo, K. \& Martuccelli, D. (2012). Desafíos comunes: retrato de la sociedad chilena y sus individuos. Tomo II. Santiago, Chile: LOM.

Araujo, K. \& Martuccelli, D. (2014). Beyond institutional individualism: Agentic individualism and the individuation process in Chilean society. Current Sociology, 62, 24-40. https://doi.org/10.1177/0011392113512496

Arfuch, L. (2010). El espacio biográfico: dilemas de la subjetividad contemporánea (3a ed.). Buenos Aires, Argentina: Fondo de Cultura Económica.

Bardin, L. (1977/2002). Análisis de contenido (3ª ed.; C. Suárez, Trad.; Título original: L'analyse de contenu). Madrid, España: Akal

Barrientos Delgado, J. (2015). Violencia homofóbica en América Latina y Chile. Santiago, Chile: El Buen Aire.

Barrientos, J. E. \& Páez, D. (2006). Psychosocial variables of sexual satisfaction in Chile. Journal of Sex \& Marital Therapy, 32, 351368. https://doi.org/10.1080/00926230600834695

Beck, U. \& Beck-Gernsheim, E. (1990/2003). El normal caos del amor: las nuevas formas de la relación amorosa (D. Schmitz, Trad.; Título original: Das ganz normale Chaos der Liebe). Barcelona, España: El Roure/Paidós.

Benavente R., M. C. \& Vergara P., C. (2006). Sexualidad en hombres y mujeres: diversidad de miradas. Santiago, Chile: Facultad Latinoamericana de Ciencias Sociales.

Bertaux, D. (1980/1999). El enfoque biográfico: su validez metodológica, sus potencialidades (TCU 0113020 de la Universidad de Costa Rica, Trad.; Título original: L'approche biographique : Sa validité méthodologique, ses potentialités). Proposiciones, Ediciones Sur, 29, 1-23.

Besoain Arrau, C., Sharim Kovalskys, D., Carmona Sepúlveda, M., Bravo Vidal, D. \& Barrientos Delgado, J. (2017). Sin conflicto y sin deseo: las tensiones de la individualización en la experiencia de pareja de jóvenes chilenos. Revista CES Psicología, 10(1), $109-128$. https://doi.org/10.21615/cesp.10.1.9

Bonan, C. (2005). Reflexividade, sexualidade e reprodução: encruzilhadas das modernidades latino-americanas [Reflexividad, sexualidad y reproducción: encrucijadas de las modernidades latinoamericanas]. Iberoamericana, 18, 89-107.

Brown, J. (2005). The compelling nature of romantic love: A psychosocial perspective. Psychoanalysis, Culture \& Society, 10 , 23-43. https://doi.org/10.1057/palgrave.pcs.2100039

Caillaud, S. \& Kalampalikis, N. (2013). Focus groups and ecological practices: A psychosocial approach. Qualitative Research in Psychology, 10, 382-401. https://doi.org/10.1080/14780887.2012.674176

Cornejo, M. (2006). El enfoque biográfico: trayectorias, desarrollos teóricos y perspectivas. Psykhe, 15(1), 95-106. https://doi.org/10.4067/S071822282006000100326

Cornejo, M., Mendoza, F. \& Rojas, R. C. (2008). La investigación con relatos de vida: pistas y opciones del diseño metodológico. Psykhe, 17(1), 29-39. https://doi.org/10.4067/S0718-222820080001000189

Correa L., R. (1999). La aproximación biográfica como una opción epistemológica, ética y metodológica. Proposiciones, Ediciones Sur, 29, 1-9.

Costa, S. (2006). ¿Amores fáciles? Romanticismo y consumo en la modernidad tardía. Revista Mexicana de Sociología, 68, 761-782.

Cruz Sierra, S. (2011). Sentido y práctica de la intimidad masculina. Una mirada desde los hombres. Sociológica, 26(73), $183-207$.

de Villers Grand Champs, G. (1993/1999). La historia de vida como método clínico (M. Labarca Sanseloni, Trad.; Título original: Histoire de vie comme méthode clinique). Proposiciones, Ediciones Sur, 29, 1-12.

Digneffe, F. (1995). De l'individuel au social : L'approche biographique [De lo individual a lo social: el enfoque biográfico]. En L. Albarello, F. Digneffe, J. -P. Hiernaux, C. Maroy, D. Ruquoy \& P. de Saint-Georges, Pratiques et méthodes de recherche en sciences sociales [Prácticas y métodos de investigación en ciencias sociales] (pp. 145-173). Paris, Francia: Armand Colin.

Ehrenberg, A. (1998/2000). La fatiga de ser uno mismo: depresión y sociedad (R. C. Paredes, Trad.; Título original: La fatigue d'ê soi : Dépression et société). Buenos Aires, Argentina: Nueva Visión.

Etherington, K. (2004). Becoming a reflexive researcher: Using our selves in research. London, Reino Unido: Jessica Kingsley.

Ferrarotti, F. (1981/2013). Histoire et histoires de vie : La méthode biographique dans les sciences sociales [Historia e historias de vida: el método biográfico en las ciencias sociales] (M. Modak, Trad.; Título original: Storia e storie di vita). Paris, Francia: Téraèdre.

Flick, U. (2002/2004). Introducción a la investigación cualitativa (J. Torres Santomé, Trad.; Título original: Qualitative Sozialforschung). Madrid, España: Morata.

Frank, A., Clough, P. T. \& Seidman, S. (Eds.) (2013). Intimacies: A new world of relational life. New York, NY: Routledge.

Giddens, A. (1992). The transformation of intimacy: Sexuality, love \& eroticism in modern societies. Cambridge, Reino Unido: Polity Press.

Goldner, V. (2004). Attachment and eros: Opposed or synergistic? Psychoanalytic Dialogues, 14, 381-396. https://doi.org/10.1080/ 10481881409348793

Goldner, V. (2007). "Let's do it again": Further reflections on eros and attachment. Psychoanalytic Dialogues, 16, 619-637. https://doi.org/10.1080/10481880701357248

Güell, P., Peters, T. \& Morales, R. (2012). Individuación y consumo cultural: las afinidades electivas. En P. Güell \& T. Peters (Eds.), La trama social de las prácticas culturales: sociedad y subjetividad en el consumo cultural de los chilenos (pp. 21-49). Santiago, Chile: Universidad Alberto Hurtado. 
Guevara Ruiseñor, E. S. (2005). Intimidad y modernidad. Precisiones conceptuales y su pertinencia para el caso de México. Estudios Sociológicos, 23, 857-877.

Guevara Ruiseñor, E. S. (2007). Intimidad. Los vínculos amorosos y los desafíos de la modernidad. En G. Zabludovsky Kuper (Coord.), Sociología y cambio conceptual: de la burocracia y las normas al cuerpo y la intimidad (pp. 248-290). México DF, México: Siglo XXI/Universidad Nacional Autónoma de México/Universidad Autónoma Metropolitana, Azcapotzalco.

ICCOM Investigación de Mercado (2005). Descripción básica de los niveles sociales: hogares urbanos Región Metropolitana - 2005. Santiago, Chile: Autor. Extraído de https://slidedoc.es/descripcion-basica-de-los-niveles-sociales-hogares-urbanos-regionmetropolitana-prohibida-su-reproduccion-pdf

Illouz, E. (2006/2007). Intimidades congeladas: las emociones en el capitalismo [J, Ibarburu, Trad.; Título original: Cold intimacies: The making of emotional capitalism]. Buenos Aires, Argentina: Katz.

Illouz, E. (2011/2012). Por qué duele el amor: una explicación sociológica. [M. V. Rodil, Trad.; Título original: Warum Liebe weh tut]. Buenos Aires, Argentina: Katz.

Lainé, A. (1998). Faire de sa vie une histoire. Théories et pratiques de l'histoire de vie en formation [Hacer de la vida una historia. Teoría y práctica del relato de vida en formación]. Paris, Francia: Desclé de Brouwer.

Legrand, M. (1993). L'approche biographique: Théorie, clinique [El enfoque biográfico: teoría, clínica]. Paris, Francia: Desclée de Brouwer.

López Mondéjar, L. (2003). Amores en fuga. Revista de la Asociación Española de Neuropsiquiatría, 23(87), 2501-2521. https://doi.org/10.4321/S0211-57352003000300007

Luepnitz, D. A. (2002). Schopenhauer's porcupines: Intimacy and its dilemmas. Five stories of psychotherapy. New York, NY: Basic Books.

Martuccelli, D. (2010). La individuación como macrosociología de la sociedad singularista. Persona y Sociedad, 24(3), 9-29.

Mitchell, S. A. (1997). Psychoanalysis and the degradation of romance. Psychoanalytic Dialogues, 7 , 23-41. https://doi.org/10.1080/10481889709539165

Mitchell, S. A. (2002). Can love last? The fate of romance over time. New York, NY: Norton.

Molitor, M. (1990). L'herméneutique collective [La hermenéutica colectiva]. En J. Remy \& D. Ruquoy (Dirs.), Méthodes d'analyse de contenu et sociologie [Métodos de análisis de contenido y sociología] (pp. 19-35). Bruxelles, Bélgica: Facultés Universitaires Saint- Louis.

Morgan, D. L. \& Spanish, M. T. (1984). Focus groups: A new tool for qualitative research. Qualitative Sociology, 7 , $253-270$. https://doi.org/10.1007/BF00987314

Patton, M. Q. (2002). Qualitative research \& evaluation methods (3a ed.). Thousand Oaks, CA: SAGE.

Programa de Naciones Unidas para el Desarrollo (2002). Desarrollo humano en Chile. Vol. 2 Nosotros los chilenos: un desafío cultural. Santiago, Chile: LOM.

Programa de Naciones Unidas para el Desarrollo (2015). Desarrollo humano en Chile: los tiempos de la politización. Santiago, Chile: Autor.

Rabiee, F. (2004). Focus-group interview and data analysis. Proceedings of the Nutrition Society, 63, 655-660. https://doi.org/10.1079/PNS2004399

Sharim, D. (2001). Los relatos de vida como herramienta para la investigación y formación clínica. Psykhe, 10(2), 71-76.

Sharim Kovalskys, D. (2005). La identidad de género en tiempos de cambio: una aproximación desde los relatos de vida. Psykhe, 14(2), 19-32. https://doi.org/10.4067/S0718-22282005000200002

Sharim, D., Araya, C., Carmona, M. \& Riquelme, P. (2011). Relatos de historias de pareja en el Chile actual: la intimidad como un monólogo colectivo. Psicologia em Estudo, 16, 347-358. https://doi.org/10.1590/S1413-73722011000300002

Sharim, D., Kovalskys, J., Morales, G. \& Cornejo, M. (2011). Trauma psicosocial y memoria: diseño de un dispositivo biográfico para investigar el impacto de la Comisión de Prisión Política y Tortura en Chile. Revista de Estudios Sociales, 40, 81-88. https://doi.org/10.7440/res40.2011.08

Starks, H. \& Trinidad, S. B. (2007). Choose your method: A comparison of phenomenology, discourse analysis, and grounded theory. Qualitative Health Research, 17, 1372-1380. https://doi.org/10.1177/1049732307307031

Tong, A., Sainsbury, P. \& Craig, J. (2007). Consolidated criteria for reporting qualitative research (COREQ): A 32 -item checklist for interviews and focus groups. International Journal for Quality in Health Care, 19, 349-357. https://doi.org/10.1093/intqhc/mzm042

Valdés, T., Gysling, J. \& Benavente, M. C. (1999). El poder en la pareja, la sexualidad y la reproducción. Mujeres de Santiago. Santiago, Chile: Facultad Latinoamericana de Ciencias Sociales.

Valsiner, J. (2009). Integrating psychology within the globalizing world: A requiem to the post-modernist experiment with Wissenschaft. Integrative Psychological \& Behavioral Science, 43, 1-21. https://doi.org/10.1007/s12124-009-9087-x

Yopo, M. (2013). Individualización en Chile: individuo y sociedad en las transformaciones culturales recientes. Psicoperspectivas, 12(2), 4-15. https://doi.org/10.5027/PSICOPERSPECTIVAS-VOL13-ISSUE2-FULLTEXT-254

Fecha de recepción: Marzo de 2016.

Fecha de aceptación: Junio de 2017. 